\title{
Big Data in Engineering: Opportunities, Challenges, and Applications
}

\author{
Kai-Uwe Sattler $^{(\bowtie)}$ \\ Department of Computer Science and Automation, \\ TU Ilmenau, Ilmenau, Germany \\ kus@tu-ilmenau.de
}

\begin{abstract}
Modern data management technology opens great opportunities for handling and analyzing huge datasets in many application domains. This is particularly interesting for engineering fields where the task of leveraging data from measurements and process monitoring plays an important role. However, handling this massive amount of data and making sense out of this data often requires an interdisciplinary approach combining expertise from data management experts, data scientists, and domain experts. In this talk we discuss this topic from a database technology perspective. We present opportunities in selected domains of engineering, identify challenges, and present technical solutions and trends in engineering data management. Finally, we discuss some application examples we currently try to address in our work.
\end{abstract}

